\title{
New Methodology for the Deoxygenative Difluoromethylenation of Aldehydes and Ketones; Unexpected Formation of Tetrafluorocyclopropanes
}

\author{
Ireneusz Nowak and Morris J. Robins* \\ Department of Chemistry and Biochemistry, Brigham Young University, Provo, Utah 84602- \\ 5700 \\ morris_robins@byu.edu
}

\section{Supporting Information}

Pages S2-S6: Experimental procedures and characterization data

Pages S7-S21: ${ }^{1} \mathrm{H}$ and ${ }^{13} \mathrm{C}$ NMR spectra of $\mathbf{2 a - j}, \mathbf{3 a}, \mathbf{b}, \mathbf{d}, \mathbf{g}$, and 1,1,2,2-tetrafluoro-3-(4-bromo-3nitrophenyl)-3-methylcyclopropane

Page S22: Structure of 1,1,2,2-tetrafluoro-3-(4-bromo-3-nitrophenyl)-3-methylcyclopropane 


\section{Experimental Section}

CAUTION! Organomercury compounds are extremely toxic. Avoid skin contact, use heavy-duty protective gloves, and use all other safety precautions for the handling and disposal of highly toxic materials!

General: Uncorrected melting points were determined with a Hoover capillary apparatus. ${ }^{1} \mathrm{H}(500 \mathrm{MHz}),{ }^{13} \mathrm{C}(125 \mathrm{MHz})$, and ${ }^{19} \mathrm{~F}(288 \mathrm{MHz})$ NMR spectra were recorded in $\mathrm{CDCl}_{3}$ unless noted. Electron impact (EI) mass spectra were obtained at $70 \mathrm{eV}$. High-resolution mass spectra (HRMS) were determined with EI ionization. All chemicals and solvents were of reagent quality.

Synthesis of Bis(trifluoromethyl)mercury: A mixture of $\mathrm{HgO}(22.0 \mathrm{~g}, 102 \mathrm{mmol}), \mathrm{H}_{2} \mathrm{O}$ $(5 \mathrm{~mL})$ and TFA $(18.2 \mathrm{~mL}, 27.0 \mathrm{~g}, 237 \mathrm{mmol})$ was heated gently in a closed flask (500 mL) until a clear, viscous solution of $\mathrm{Hg}\left(\mathrm{O}_{2} \mathrm{CCF}_{3}\right)_{2}$ was formed. The flask was then placed in an oil bath and heated at $110{ }^{\circ} \mathrm{C}$ under vacuum for $2 \mathrm{~h}$ to give a crystalline solid product. [Anhydrous $\mathrm{K}_{2} \mathrm{CO}_{3}$ (33.0 g, $239 \mathrm{mmol}$ ) was heated in a crucible dish for $30 \mathrm{~min}$ over a hot flame (Bunsen burner). The resulting solid was transferred to a mortar and ground to a fine powder while hot $\left(>100{ }^{\circ} \mathrm{C}\right)$.] The freshly dried $\mathrm{K}_{2} \mathrm{CO}_{3}$ was added to the flask containing the dried crystalline $\mathrm{Hg}\left(\mathrm{O}_{2} \mathrm{CCF}_{3}\right)_{2}$. The flask was fitted with a cold-finger condenser, a vacuum pump was attached, and the flask was placed in an oil bath. The temperature was increased to $150{ }^{\circ} \mathrm{C}$ while the system was kept under vacuum. When the oil-bath temperature reached $150{ }^{\circ} \mathrm{C}$, the condenser reservoir was filled with Dry Ice/ $\mathrm{Me}_{2} \mathrm{CO}$. Smooth decomposition occurred at $180-205{ }^{\circ} \mathrm{C}$ during $2-3 \mathrm{~h}$, and the product $\left(\mathrm{CF}_{3}\right)_{2} \mathrm{Hg}$ sublimed and was deposited on the lower inner surface of the condenser. The flask was removed from the oil bath and allowed to cool to ambient temperature. The vacuum was removed, and the solid product was scraped from the inner surface of the condenser. The remaining crystalline film was washed out with benzene. The solid product was dissolved in the benzene solution, and a minor amount of contaminating mercury metal was removed by filtration though a small column of silica gel. Evaporation of benzene gave bis(trifluoromethyl)mercury (typical yield: $18.9 \mathrm{~g}, 55 \%$ ).

Synthesis of Compounds 2. General Procedure A: Powdered NaI (2.97 g, 19.8 mmol) in a flask $(250 \mathrm{~mL})$ equipped with a Teflon valve was stirred and heated in an oil bath at $170{ }^{\circ} \mathrm{C}$ for $1 \mathrm{~h}$ under vacuum, and the flask and contents were allowed to cool to ambient temperature. A solution of the carbonyl compound $(1.65 \mathrm{mmol}),\left(\mathrm{CF}_{3}\right)_{2} \mathrm{Hg}(1.68 \mathrm{~g}, 4.95 \mathrm{mmol})$, and $\mathrm{Bu}_{3} \mathrm{P}(1.23$ 
$\mathrm{mL}, 1.00 \mathrm{~g}, 4.95 \mathrm{mmol})$ in dried THF $(15 \mathrm{~mL})$ was injected through a septum under an atmosphere of $\mathrm{N}_{2}$. The reaction mixture was heated in an oil bath at $70{ }^{\circ} \mathrm{C}$ for $2 \mathrm{~h}$, and cooled to ambient temperature. Volatiles were concentrated, and the mixture was deposited on a column of silica gel. Elution $\left(\mathrm{CH}_{2} \mathrm{Cl}_{2} /\right.$ hexanes, $\left.1: 3\right)$ gave the crude product (analyzed by $\left.{ }^{19} \mathrm{~F} \mathrm{NMR}\right)$. This material was chromatographed on silica gel (hexanes for: $\mathbf{2 a}-\mathbf{h}, \mathbf{3 a}, \mathbf{b}, \mathbf{d}, \mathbf{g}),($ EtOAc/hexanes, 1:50 for: $\mathbf{2 j}$ ), or (EtOAc/hexanes, 1:2 for: $\mathbf{2 i}$ ). The difluoromethylene compounds $\mathbf{2}$ were eluted first, followed by mixtures of $\mathbf{2}$ and 3. Purified $\mathbf{3}$ was obtained by recrystallization of the mixture of $\mathbf{2}$ and 3 from $\mathrm{MeOH}$. A suitably purified sample of $\mathbf{2}$ was obtained after evaporation of solvents for all products except $\mathbf{2} \mathbf{i}$, which was dissolved in $\mathrm{MeOH}(3 \mathrm{~mL})$ and treated with concentrated $\mathrm{HCl} / \mathrm{H}_{2} \mathrm{O}(0.2 \mathrm{~mL})$ (with stirring). Volatiles were evaporated in vacuo, and $\mathbf{2} \mathbf{i} \cdot \mathrm{HCl}$ was obtained by silica gel chromatography (EtOAc then $\mathrm{MeOH})$. The methanolic fractions were concentrated, neutralized with $\mathrm{NaHCO}_{3} / \mathrm{H}_{2} \mathrm{O}$, and extracted with EtOAc. The combined organic phase was concentrated and chromatographed (silica; EtOAc/hexanes, 1:5) to give $2 \mathbf{i}$ as a pale-yellow oil.

Synthesis of Tetrafluorocyclopropanes 3a,b,d,g from 2a,b,d,g. General Procedure B: A solution of $2 \mathrm{a}(0.56 \mathrm{~g}, 3.11 \mathrm{mmol})$, dried NaI (1.48 g, $9.87 \mathrm{mmol}),\left(\mathrm{CF}_{3}\right)_{2} \mathrm{Hg}(0.84 \mathrm{~g}, 2.48$ $\mathrm{mmol})$, and $\mathrm{Ph}_{3} \mathrm{P}(0.65 \mathrm{~g}, 4.48 \mathrm{mmol})$ in THF $(15 \mathrm{~mL})$ was stirred in an oil bath at $70{ }^{\circ} \mathrm{C}$ for $2 \mathrm{~h}$. The resulting mixture of $\mathbf{2 a}$ and $\mathbf{3 a}$ was isolated as described for the synthesis of $\mathbf{2 a}$. This mixture was treated successively by procedure B until the ${ }^{19} \mathrm{~F}$ NMR spectrum indicated minor traces of starting $\mathbf{2 a}$. The yield of purified $\mathbf{3 a}(0.53 \mathrm{~g})$ was $95 \%$.

\section{Synthesis of 1,1,2,2-Tetrafluoro-3-(4-bromo-3-nitrophenyl)-3-methylcyclopropane:}

Treatment of 4-bromoacetophenone $(0.90 \mathrm{~g}, 4.52 \mathrm{mmol})$ and triphenylphosphine by general procedure A (without isolation of the difluoromethylene intermediate) followed by two cycles of general procedure B gave 1,1,2,2-tetrafluoro-3-(4-bromophenyl)-3-methylcyclopropane (0.7 g, $54 \%)$. Cold $\left(0{ }^{\circ} \mathrm{C}\right)$ fuming nitric acid $(1.5 \mathrm{~mL})$ was added to this product, and the mixture was stirred for $30 \mathrm{~min}$. The mixture was poured on to ice and extracted with EtOAc $(2 \times 15 \mathrm{~mL})$. Volatiles were concentrated, and chromatography (EtOAc/hexanes, 1:6) gave material (0.66 g, $84 \%$ ) that was purified (recrystallization from $\mathrm{MeOH}$ ) to give crystals for X-ray analysis.

\section{Characterization Data:}

1-(Difluoromethylene)-1,2,3,4-tetrahydronaphthalene (2a). Colorless oil: ${ }^{1} \mathrm{H}$ NMR $\delta$ 1.74-1.82 (m, 2H), 2.38-2.46 (m, 2H), 2.70-2.76 (m, 2H), 7.04-7.15 (m, 3H), 7.55 (d, J = 7.8 
$\mathrm{Hz}, 1 \mathrm{H}) ;{ }^{13} \mathrm{C}$ NMR $\delta 22.3,23.3,30.4,88.3(\mathrm{dd}, J=8.4,23.7 \mathrm{~Hz}), 126.1,126.6,127.1,127.2$, 129.0, $137.6(\mathrm{~d}, J=6.1 \mathrm{~Hz}), 152.9(\mathrm{dd}, J=284.6,296.0 \mathrm{~Hz}) ;{ }^{19} \mathrm{~F}$ NMR $\delta 88.2(\mathrm{~d}, J=42.7 \mathrm{~Hz}$, 1F), $88.6(\mathrm{~d}, J=42.7 \mathrm{~Hz}, 1 \mathrm{~F})$; MS $m / z 180\left(100 \%, \mathrm{M}^{+}\right), 159,147,129,128,115 ;$ HMRS Calcd for $\mathrm{C}_{11} \mathrm{H}_{10} \mathrm{~F}_{2}$ : 180.0751; Found: 180.0748 .

\section{1,1,2,2-Tetrafluoro-3,3-[1-(1,2,3,4-tetrahydro)naphthylidene]cyclopropane (3a).}

Colorless oil: ${ }^{1} \mathrm{H}$ NMR $\delta$ 1.79-1.83 (m, 2H), 1.97-2.02 (m, 2H), 2.77-2.81 (m, 2H), 7.10-7.20 (m, 3H), 7.31-7.35 (m, 1H); ${ }^{13} \mathrm{C}$ NMR $\delta 21.0,22.2(\mathrm{t}, J=7.6 \mathrm{~Hz}), 29.8,39.1$ (q, $\left.J=10.3 \mathrm{~Hz}\right)$, $108.2(\mathrm{ddt}, J=311.3,315.1,10.3 \mathrm{~Hz}), 125.9,126.2$, $127.7,127.8$ (t, $J=6.9 \mathrm{~Hz}), 129.4$, 139.1; ${ }^{19} \mathrm{~F}$ NMR $\delta 150.6(\mathrm{~d}, J=176.2 \mathrm{~Hz}, 2 \mathrm{~F}), 152.2(\mathrm{~d}, J=176.2 \mathrm{~Hz}, 2 \mathrm{~F}) ; \mathrm{MS} m / z 230\left(30 \%, \mathrm{M}^{+}\right), 202$ $\left(100 \%, \mathrm{M}^{+}-28\right), 180,129$; HMRS Calcd for $\mathrm{C}_{12} \mathrm{H}_{10} \mathrm{~F}_{4}$ : 230.0719; Found: 230.0724.

(4,4-Difluoro-3-methyl-3-buten-1-yl)benzene (2b). Colorless oil: ${ }^{1} \mathrm{H}$ NMR $\delta 1.54$ (t, $J=$ $3.2 \mathrm{~Hz}, 3 \mathrm{H}), 2.21-2.26(\mathrm{~m}, 2 \mathrm{H}), 2.66(\mathrm{t}, J=7.7 \mathrm{~Hz}, 2 \mathrm{H}), 7.12-7.27(\mathrm{~m}, 5 \mathrm{H}) ;{ }^{13} \mathrm{C}$ NMR $\delta 11.8$, $30.2,33.6,84.2(\mathrm{t}, J=18.7 \mathrm{~Hz}), 126.0,128.3,128.4,141.2,153.0(\mathrm{t}, J=281.9 \mathrm{~Hz}) ;{ }^{19} \mathrm{~F} \mathrm{NMR} \delta$ $96.7(\mathrm{~d}, J=57.7 \mathrm{~Hz}, 1 \mathrm{~F}), 97.1(\mathrm{~d}, J=57.7 \mathrm{~Hz}, 1 \mathrm{~F}) ; \mathrm{MS} m / z .182\left(20 \%, \mathrm{M}^{+}\right), 115,91\left(100 \%, \mathrm{M}^{+}\right.$ - 91); HMRS Calcd for $\mathrm{C}_{11} \mathrm{H}_{12} \mathrm{~F}_{2}$ : 182.0907; Found: 182.0905.

1,1,2,2-Tetrafluoro-3-(2-phenylethyl)-3-methylcyclopropane (3b). Colorless oil: ${ }^{1} \mathrm{H}$ NMR $\delta 1.30-1.32(\mathrm{~m}, 3 \mathrm{H}), 1.87-1.98(\mathrm{~m}, 2 \mathrm{H}), 2.69-2.73(\mathrm{~m}, 2 \mathrm{H}), 7.17-7.32(\mathrm{~m}, 5 \mathrm{H}) ;{ }^{13} \mathrm{C}$ NMR $\delta 8.8(\mathrm{t}, J=7.6 \mathrm{~Hz}), 27.9(\mathrm{t}, J=5.6 \mathrm{~Hz}), 32.2,34.5(\mathrm{q}, J=10.7 \mathrm{~Hz}), 107.8(\mathrm{tt}, J=310.1,11.8$ $\mathrm{Hz}), 126.3,128.2,128.6,140.6 ;{ }^{19} \mathrm{~F}$ NMR $\delta 152.3(\mathrm{dm}, J=177.2 \mathrm{~Hz}, 2 \mathrm{~F}), 154.1(\mathrm{dm}, J=\sim 180$ $\mathrm{Hz}, 2 \mathrm{~F}) ; \mathrm{MS} m / z 232\left(10 \%, \mathrm{M}^{+}\right), 140,131\left(100 \%, \mathrm{M}^{+}-141\right)$; HMRS Calcd for $\mathrm{C}_{12} \mathrm{H}_{12} \mathrm{~F}_{4}$ : 232.0875; Found: 232.0882.

1,1-Difluoro-2,2-diphenylethene (2c). Colorless oil: ${ }^{1} \mathrm{H}$ NMR $\delta 7.16-7.26(\mathrm{~m}, 10 \mathrm{H}) ;{ }^{13} \mathrm{C}$ NMR $\delta 96.3(\mathrm{t}, J=17.9 \mathrm{~Hz}), 127.5,128.3,129.5,134.3,153.7(\mathrm{t}, J=293.4 \mathrm{~Hz}) ;{ }^{19} \mathrm{~F}$ NMR $\delta 88.3$ (s, 2F); MS m/z $216\left(65 \%, \mathrm{M}^{+}\right), 165\left(100 \%,\left[\mathrm{M}^{+}-51\right]\right)$; HRMS Calcd for $\mathrm{C}_{14} \mathrm{H}_{10} \mathrm{~F}_{2}: 216.0751$; Found: 216.0751.

\section{1,1,2,2-Tetrafluoro-3,3-diphenylcyclopropane (3c). ${ }^{19} \mathrm{~F}$ NMR $\delta 142.4$ (s, 4F).}

2-(1,1-Difluoroprop-1-en-2-yl)naphthalene (2d). Colorless oil: ${ }^{1} \mathrm{H}$ NMR $\delta 2.02$ (t, $J=$ $3.4 \mathrm{~Hz}, 3 \mathrm{H}), 7.40-7.49(\mathrm{~m}, 3 \mathrm{H}), 7.73-7.78(\mathrm{~m}, 4 \mathrm{H}) ;{ }^{13} \mathrm{C}$ NMR $\delta 13.3,87.7$ (dd, $J=13.7,21.4$ Hz), 125.5 (dd, $J=3.1,3.9 \mathrm{~Hz}), 126.0,126.2,126.4$ (t, $J=3.8 \mathrm{~Hz}), 127.5,127.89,127.92$, $132.27(\mathrm{t}, J=5.0 \mathrm{~Hz}), 132.31,133.2,153.7(\mathrm{dd}, J=285.3,290.7 \mathrm{~Hz}) ;{ }^{19} \mathrm{~F}$ NMR $\delta 90.2(\mathrm{~d}, J=$ 
42.7 Hz, 1F), 90.8 (d, J = 42.7 Hz, 1F); MS m/z 204 (100\%, M+), 183, 152; HMRS Calcd for $\mathrm{C}_{13} \mathrm{H}_{10} \mathrm{~F}_{2}:$ 204.0751, Found: 204.0757.

1,1,2,2-Tetrafluoro-3-methyl-3-(2-naphthyl)cyclopropane (3d). Crystals (MeOH): mp 70-72 ${ }^{\circ} \mathrm{C} ;{ }^{1} \mathrm{H}$ NMR $\delta 1.58-1.61(\mathrm{~m}, 3 \mathrm{H}), 7.29-7.32(\mathrm{~m}, 1 \mathrm{H}), 7.43-7.48(\mathrm{~m}, 2 \mathrm{H}), 7.74-7.82(\mathrm{~m}$, $4 \mathrm{H}) ;{ }^{13} \mathrm{C}$ NMR $\delta 15.6(\mathrm{t}, J=\sim 8 \mathrm{~Hz}), 39.9(\mathrm{q}, J=10.3 \mathrm{~Hz}), 107.2(\mathrm{tt}, J=11.8,310.5 \mathrm{~Hz}), 126.3$, 126.5, 126.6, 127.7, 127.9, 128.4, 128.8, 129.2, 132.9, 133.3; ${ }^{19} \mathrm{~F}$ NMR $\delta 145.4(\mathrm{~d}, J=173.0 \mathrm{~Hz}$, 1F); 150.9 (d, $173.0 \mathrm{~Hz}, 1 \mathrm{~F}) ; \mathrm{MS}$ m/z 254 (75\%, M+), 204, 128 (75\%, $\mathrm{M}^{+}$- 126); HMRS Calcd for $\mathrm{C}_{14} \mathrm{H}_{10} \mathrm{~F}_{4}$ : 254.0719; Found: 254.0729.

1-(2,2-Difluorovinyl)naphthalene (2e). Colorless oil: ${ }^{1} \mathrm{H}$ NMR $\delta 5.83$ (dd, $J=3.4,24.4$ $\mathrm{Hz}, 1 \mathrm{H}) ; 7.41-7.58(\mathrm{~m}, 4 \mathrm{H}), 7.74-7.93(\mathrm{~m}, 3 \mathrm{H}) ;{ }^{13} \mathrm{C} \mathrm{NMR} \delta 78.6(\mathrm{dd}, J=15.3,29.0 \mathrm{~Hz}), 123.7$, $125.4,125.9,126.3,126.4,126.5,127.9,128.6,131.4,133.6,156.6$ (dd, $J=288.4,296.0 \mathrm{~Hz})$; ${ }^{19} \mathrm{~F}$ NMR $\delta 83.7(\mathrm{~d}, J=29.9 \mathrm{~Hz}, 1 \mathrm{~F}), 87.5(\mathrm{dd}, J=23.5,29.9 \mathrm{~Hz}, 1 \mathrm{~F}) ; \mathrm{MS} m / z, 190\left(30 \%, \mathrm{M}^{+}\right)$, 170 (100\%, [ $\left.\left.\mathrm{M}^{+}-20\right]\right), 159$; HMRS Calcd for $\mathrm{C}_{12} \mathrm{H}_{8} \mathrm{~F}_{2}:$ 190.0594; Found: 190.0593.

(E)-(4,4-Difluoro-1,3-butadien-1-yl)benzene (2f). Colorless, readily polymerized oil: ${ }^{1} \mathrm{H}$ NMR $\delta 5.12(\mathrm{dd}, J=10.7,24.4 \mathrm{~Hz}, 1 \mathrm{H}), 6.46(\mathrm{~d}, J=15.6 \mathrm{~Hz}, 1 \mathrm{H}), 6.65(\mathrm{dd}, J=10.7,15.6$ $\mathrm{Hz}, 1 \mathrm{H}), 7.20-7.40(\mathrm{~m}, 5 \mathrm{H}) ;{ }^{13} \mathrm{C}$ NMR $\delta 82.8$ (dd, $\left.J=16.8,27.5 \mathrm{~Hz}\right), 117.7,126.2,127.6,128.6$, $131.2(\mathrm{dd}, J=2.8,11.4 \mathrm{~Hz}), 136.9,156.7(\mathrm{dd}, J=291.4,296.8 \mathrm{~Hz}) ;{ }^{19} \mathrm{~F}$ NMR $\delta 85.8(\mathrm{dd}, J=$ 25.7, 26.7 Hz, 1F), 87.5 (d, J = 25.7 Hz, 1F); MS m/z $166\left(20 \%, \mathrm{M}^{+}\right), 131,105,84(100 \%$, [M+ 82]); HMRS Calcd for $\mathrm{C}_{10} \mathrm{H}_{8} \mathrm{~F}_{2}$ : 166.0594; Found: 166.0591.

(E)-1,1,2,2-Tetrafluoro-3-(2-phenylethenyl)cyclopropane (3f). ${ }^{19} \mathrm{~F}$ NMR $\delta 141.2$ (dm, $J=\sim 180 \mathrm{~Hz}, 2 \mathrm{~F}), 151.4(\mathrm{~d}, J=183.6 \mathrm{~Hz}, 2 \mathrm{~F})$.

4-(1,1-Difluoro-1-propen-2-yl)biphenyl (2g). Crystals (MeOH): mp 62-64 ${ }^{\circ} \mathrm{C} ;{ }^{1} \mathrm{H}$ NMR $\delta 1.95(\mathrm{t}, J=3.4 \mathrm{~Hz}, 3 \mathrm{H}), 7.28-7.58(\mathrm{~m}, 9 \mathrm{H}) ;{ }^{13} \mathrm{C} \mathrm{NMR} \delta 13.2,87.2(\mathrm{dd}, J=13.7,22.1 \mathrm{~Hz})$, 126.95, 126.98, 127.4, 127.6, 127.8, 128.8, 133.7, 139.8, 140.5, 153.6 (dd, $J=286.1,290.7 \mathrm{~Hz}$ ); ${ }^{19} \mathrm{~F}$ NMR $\delta 90.2(\mathrm{~d}, J=43.6 \mathrm{~Hz}, 1 \mathrm{~F}), 90.6(\mathrm{~d}, J=43.7 \mathrm{~Hz}, 1 \mathrm{~F}) ; \mathrm{MS} m / z 230\left(100 \%, \mathrm{M}^{+}\right)$; HMRS Calcd for $\mathrm{C}_{15} \mathrm{H}_{12} \mathrm{~F}_{2}$ : 230.0907; Found: 230.0904 .

3-(4-Biphenyl)-1,1,2,2-tetrafluoro-3-methylcyclopropane (3g). Crystals (MeOH): mp 122-124 ${ }^{\circ} \mathrm{C} ;{ }^{1} \mathrm{H}$ NMR $\delta 1.55-1.63(\mathrm{~m}, 3 \mathrm{H}), 7.33-7.63(\mathrm{~m}, 9 \mathrm{H}) ;{ }^{13} \mathrm{C}$ NMR $\left(\mathrm{MeCO}-d_{6}\right) \delta 15.8(\mathrm{t}, J$ $=7.3 \mathrm{~Hz}), 40.9(\mathrm{q}, J=10.8 \mathrm{~Hz}), 108.3(\mathrm{tt}, J=11.7,309.2 \mathrm{~Hz}), 127.9,128.4,128.6,129.9,130.6$, 131.6, 141.1, 142.1; ${ }^{19} \mathrm{~F}$ NMR $\delta 145.3(\mathrm{dd}, J=6.4,173.0 \mathrm{~Hz}, 2 \mathrm{~F}), 150.9(\mathrm{dd}, J=6.4,173.0 \mathrm{~Hz}$, 2F); MS m/z $280\left(100 \%, \mathrm{M}^{+}\right), 265,230$; HMRS Calcd for $\mathrm{C}_{16} \mathrm{H}_{12} \mathrm{~F}_{4}$ : 280.0875; Found: 280.0872. 
(E)-1,1-Difluoro-2,4-diphenyl-1,3-butadiene (2h). Colorless, readily polymerized oil: ${ }^{1} \mathrm{H}$ NMR $\delta 6.21(\mathrm{~d}, J=16.1 \mathrm{~Hz}, 1 \mathrm{H}), 7.01(\mathrm{~d}, J=16.1 \mathrm{~Hz}, 1 \mathrm{H}), 7.17-7.46(\mathrm{~m}, 10 \mathrm{H}) ;{ }^{13} \mathrm{C} \mathrm{NMR} \delta$ $96.5(\mathrm{dd}, J=16.0,20.6 \mathrm{~Hz}), 121.1,126.3,127.6,128.1,128.57,128.59,130.3,131.06$ (d, $J=2.5$ $\mathrm{Hz}), 131.13(\mathrm{~d}, J=3.5 \mathrm{~Hz}), 136.9,154.0(\mathrm{dd}, J=293.0,298.3 \mathrm{~Hz}) ;{ }^{19} \mathrm{~F}$ NMR $\delta 88.6(\mathrm{~d}, J=23.5$ $\mathrm{Hz}, 1 \mathrm{~F}), 89.7$ (d, $J=25.6 \mathrm{~Hz}, 1 \mathrm{~F}) ; \mathrm{MS} m / z 242\left(90 \%, \mathrm{M}^{+}\right), 223$ (100\%, [M+ - 19]); HMRS Calcd for $\mathrm{C}_{16} \mathrm{H}_{12} \mathrm{~F}_{2}$ : 242.0907; Found: 242.0915.

(E)-1,1,2,2-Tetrafluoro-3-phenyl-3-(2-phenylethenyl)cyclopropane (3h). ${ }^{19} \mathrm{~F}$ NMR $\delta$ $142.1(\mathrm{~d}, J=175.2 \mathrm{~Hz}, 2 \mathrm{~F}), 147.6(\mathrm{~d}, J=177.3 \mathrm{~Hz}, 2 \mathrm{~F})$.

4-(3,3-Difluoro-2-phenyl-2-propenyl)morpholine (2i). Pale-yellow oil: ${ }^{1} \mathrm{H}$ NMR $\delta$ 2.44-2.49 (m, 4H), 3.28-3.30 (m, 2H), 3.64-3.69 (m, 4H), 7.24-7.38 (m, 3H), 7.46-7.50 (m, $2 \mathrm{H}) ;{ }^{13} \mathrm{C}$ NMR $\delta 52.9,55.7(\mathrm{~d}, J=3.9 \mathrm{~Hz}), 66.8,89.0(\mathrm{dd}, J=12.2,18.3 \mathrm{~Hz}), 127.2,128.14$, 128.17, 133.6, $155.2(\mathrm{dd}, J=289.9,294.5 \mathrm{~Hz}) ;{ }^{19} \mathrm{~F}$ NMR $\delta 88.5(\mathrm{~d}, J=36.3 \mathrm{~Hz}, 1 \mathrm{~F}), 88.9(\mathrm{~d}, J=$ $34.2 \mathrm{~Hz}, 1 \mathrm{~F})$; MS m/z $239\left(10 \%, \mathrm{M}^{+}\right), 153,100$ (100\%, $\mathrm{M}^{+}$- 139); HMRS Calcd for $\mathrm{C}_{13} \mathrm{H}_{15} \mathrm{~F}_{2} \mathrm{NO}$ : 239.1122; Found: 239.1125.

3,3-Difluoro-2-phenyl-2-propenyl benzoate (2j). Pale-yellow oil: ${ }^{1} \mathrm{H}$ NMR $\delta 5.18(\mathrm{~d}, J$ $=2.2 \mathrm{~Hz}, 2 \mathrm{H}), 7.26-7.54(\mathrm{~m}, 8 \mathrm{H}), 7.96-7.99(\mathrm{~m}, 2 \mathrm{H}) ;{ }^{13} \mathrm{C} \mathrm{NMR} \delta 61.0(\mathrm{~d}, J=6.1 \mathrm{~Hz}), 89.6(\mathrm{t}, J$ $=17.2 \mathrm{~Hz}), 127.8,128.0,128.3,128.6,129.6,129.7,131.6,133.1,155.6(\mathrm{t}, J=294.9 \mathrm{~Hz}), 166.2$; ${ }^{19} \mathrm{~F}$ NMR $\delta 85.4(\mathrm{~d}, J=25.6 \mathrm{~Hz}, 1 \mathrm{~F}), 85.7(\mathrm{~d}, J=25.6 \mathrm{~Hz}, 1 \mathrm{~F}) ; \mathrm{MS} m / z 274\left(55 \%, \mathrm{M}^{+}\right), 205,105$ (100\%, [ $\left.\left.\mathrm{M}^{+}-169\right]\right)$; HMRS Calcd for $\mathrm{C}_{16} \mathrm{H}_{12} \mathrm{~F}_{2} \mathrm{O}_{2}: 274.0805$; Found: 274.0810.

\section{1,1,2,2-Tetrafluoro-3-(4-bromo-3-nitrophenyl)-3-methylcyclopropane. Crystals} $(\mathrm{MeOH}): \operatorname{mp} 78-79{ }^{\circ} \mathrm{C} ;{ }^{1} \mathrm{H}$ NMR $\delta 1.58-1.61(\mathrm{~m}, 3 \mathrm{H}), 7.36(\mathrm{dd}, J=1.0,8.3 \mathrm{~Hz}, 1 \mathrm{H}), 7.78(\mathrm{~d}, J=$ $2.0 \mathrm{~Hz}, 1 \mathrm{H}), 7.79(\mathrm{~d}, J=8.3 \mathrm{~Hz}, 1 \mathrm{H}),{ }^{13} \mathrm{C} \mathrm{NMR} \delta 14.9(\mathrm{t}, J=\sim 6 \mathrm{~Hz}), 38.7$ (q, $\left.J=10.3 \mathrm{~Hz}\right), 106.0$ $(\mathrm{tt}, J=11.4,311.0 \mathrm{~Hz}), 114.8,126.2$, 132.6, 133.8, 135.8, 150.0; ${ }^{19} \mathrm{~F}$ NMR $\delta 144.9(\mathrm{dd}, J=4.3$, $173.0 \mathrm{~Hz}, 2 \mathrm{~F}), 150.5$ (dd, $J=4.3,173.0 \mathrm{~Hz}, 2 \mathrm{~F})$; FAB-MS $m / z 327\left(100 \%, \mathrm{M}^{+}\right)$; HMRS Calcd for $\mathrm{C}_{10} \mathrm{H}_{6} \mathrm{BrF}_{4} \mathrm{NO}_{2}$ : 326.9517; Found: 326.9520 . 

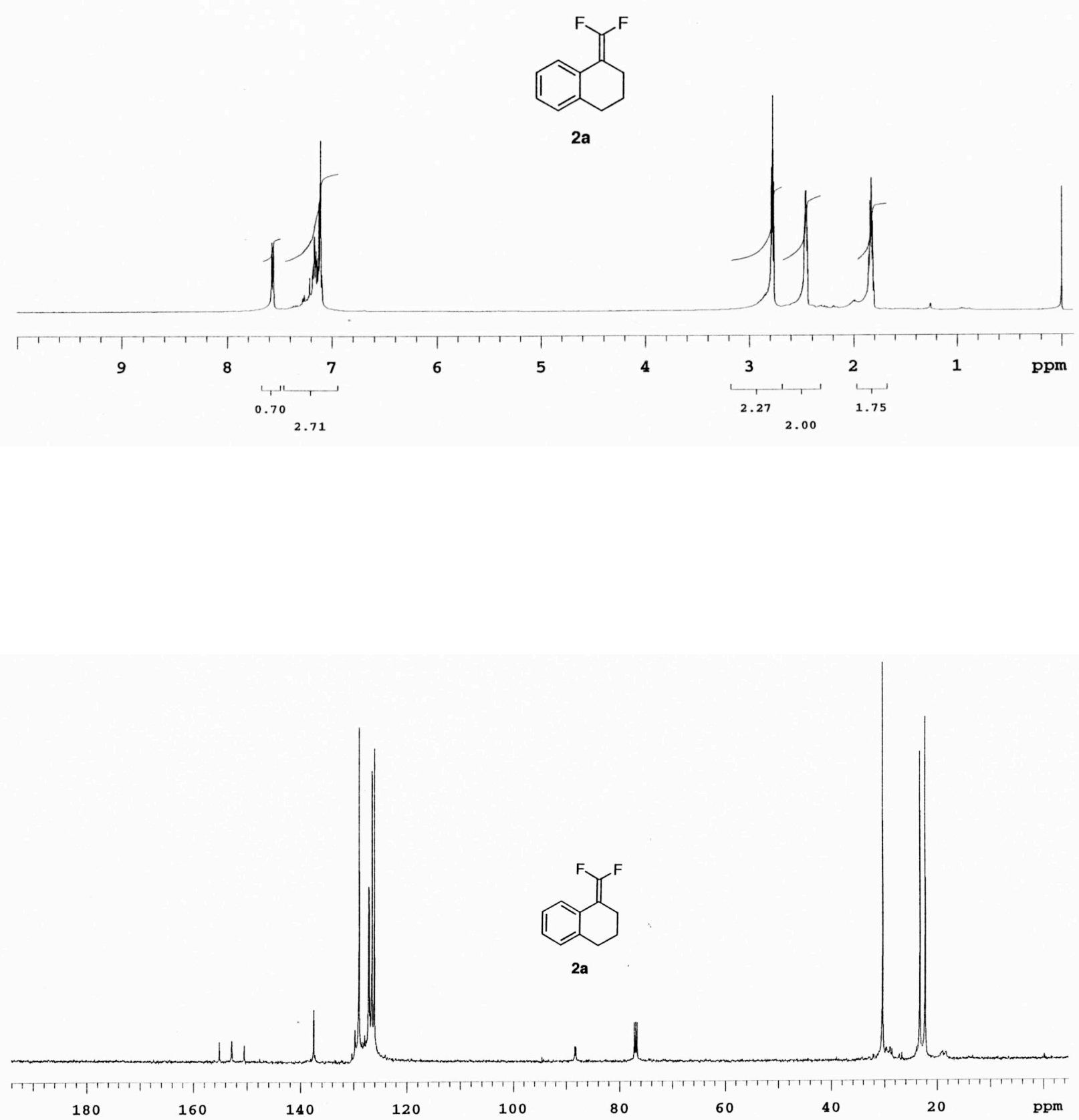
S8
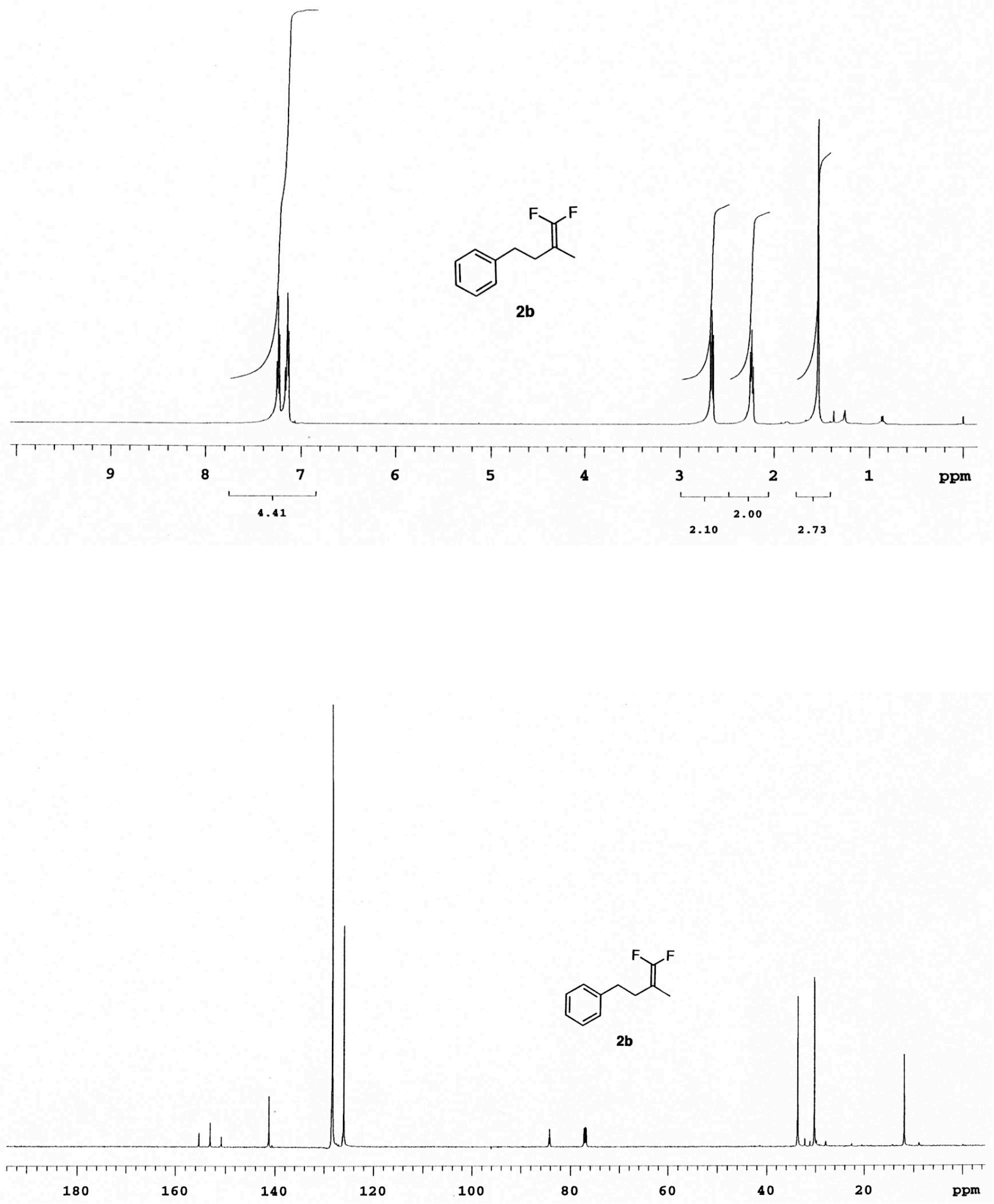

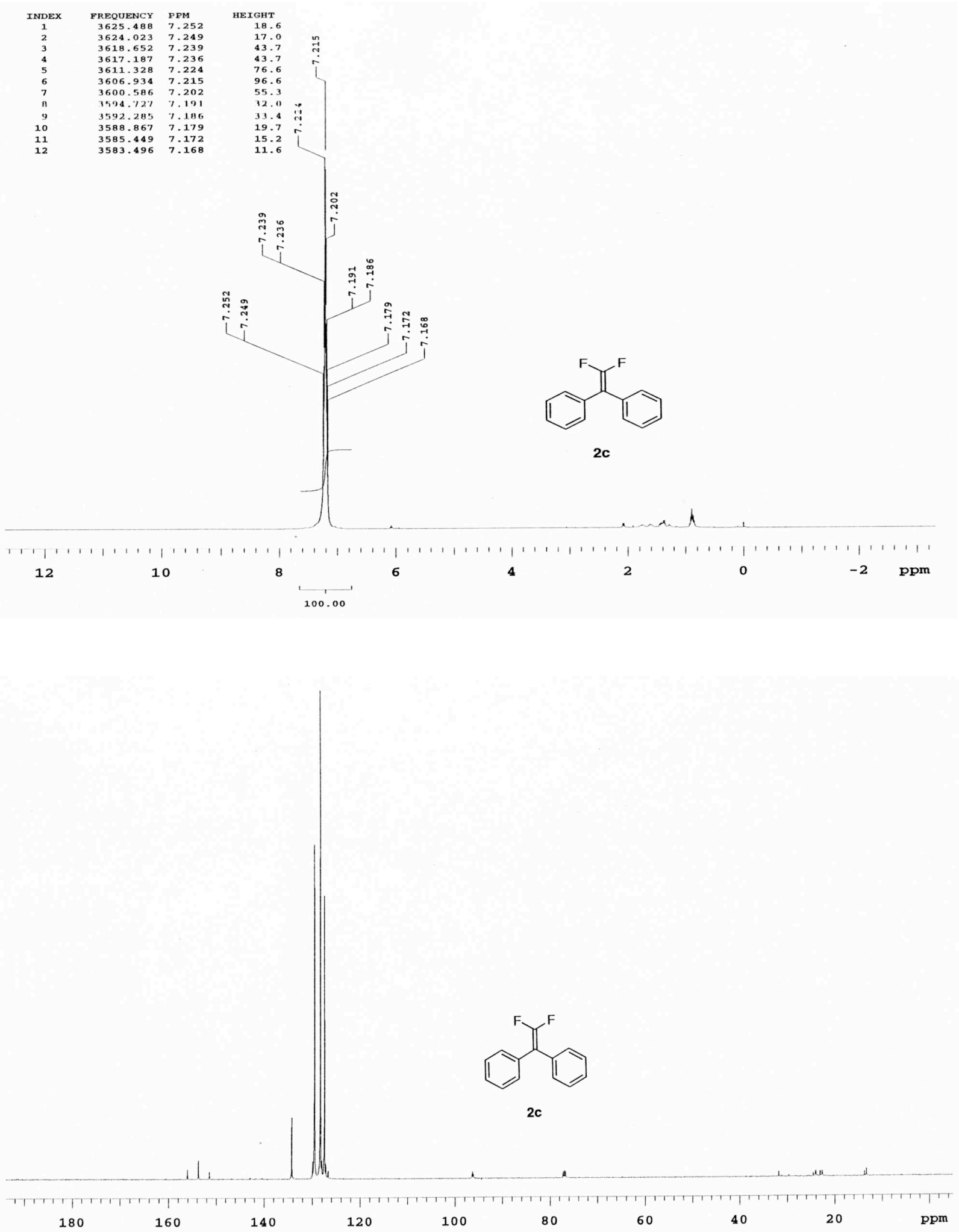

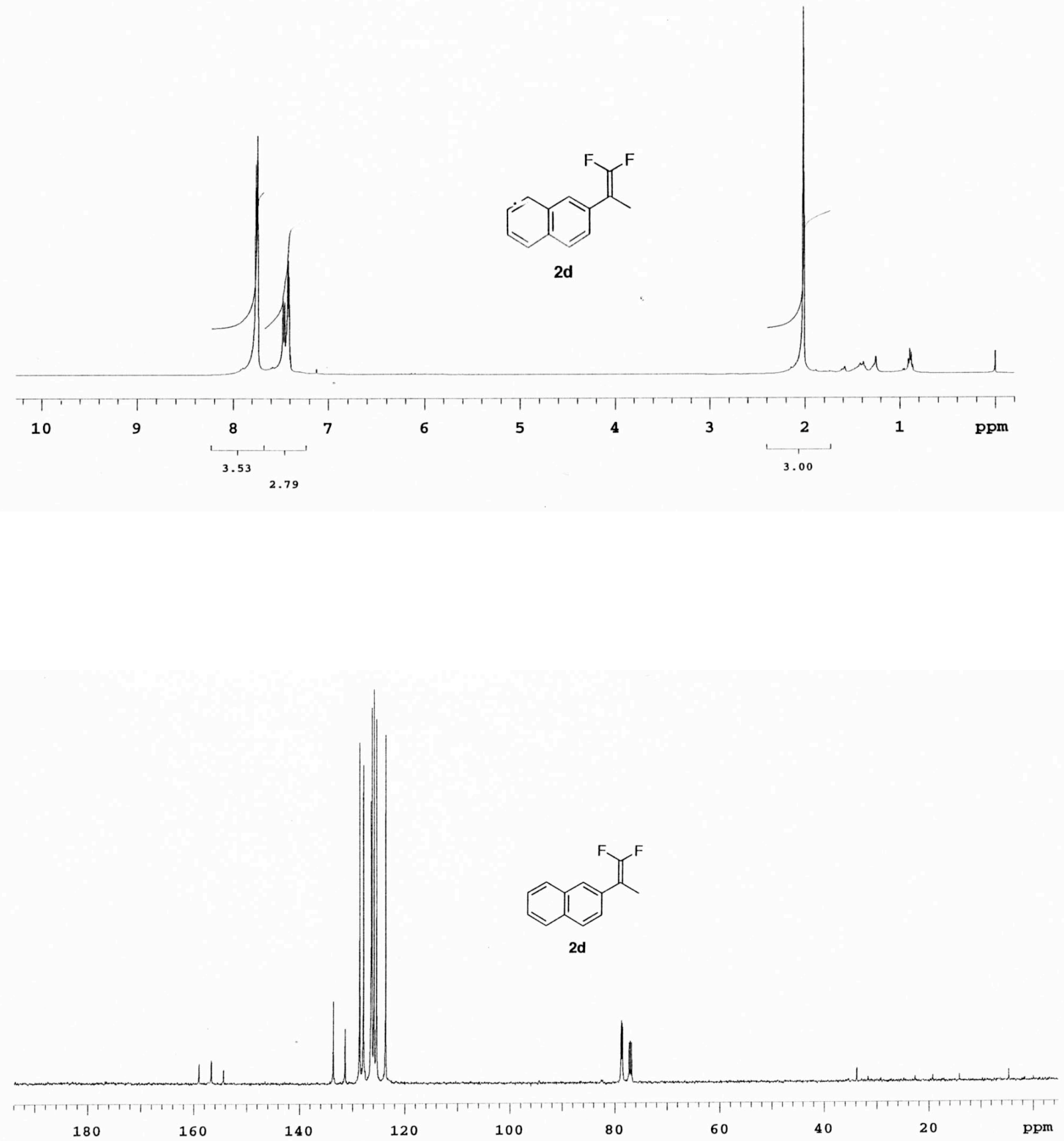

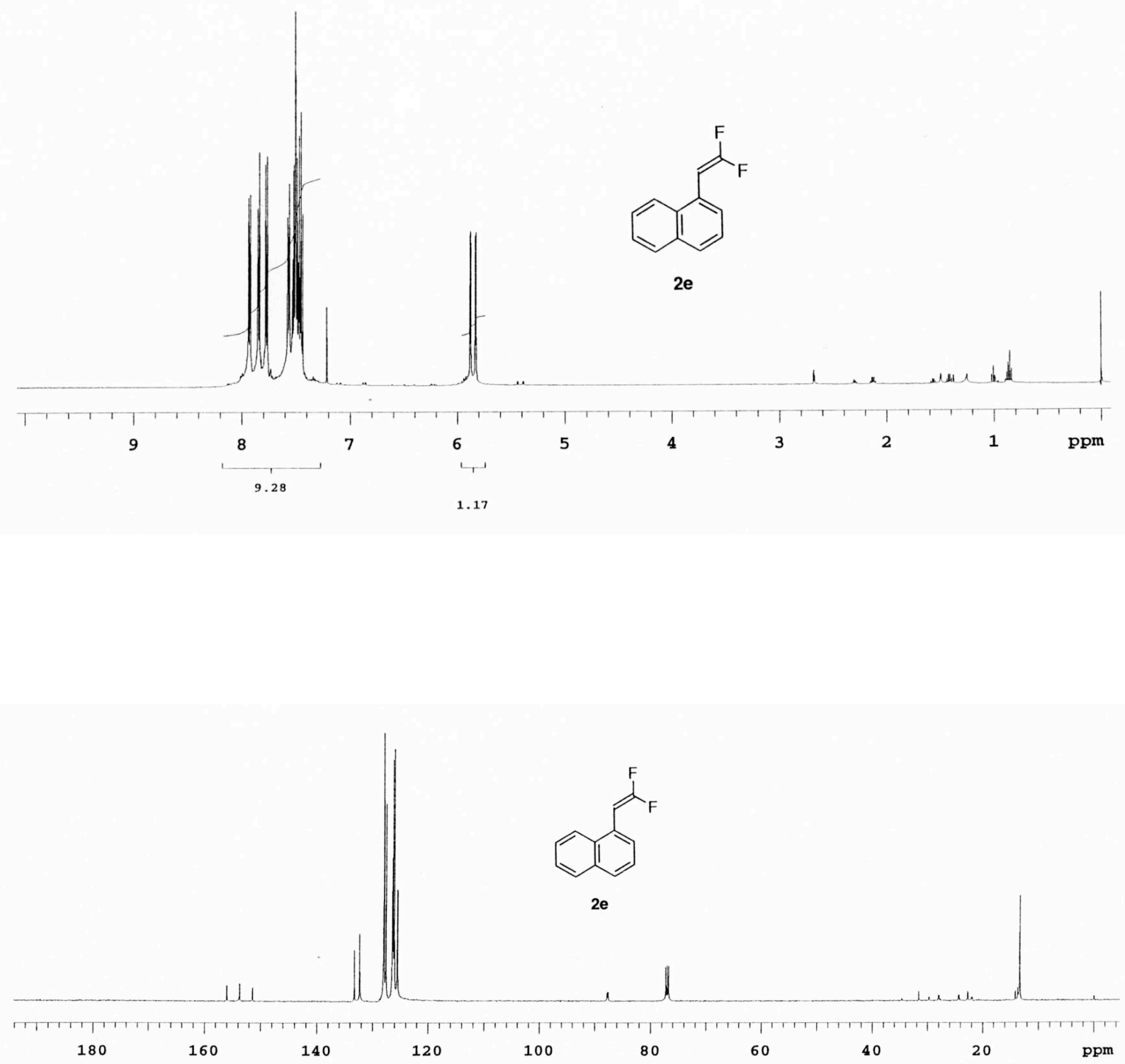

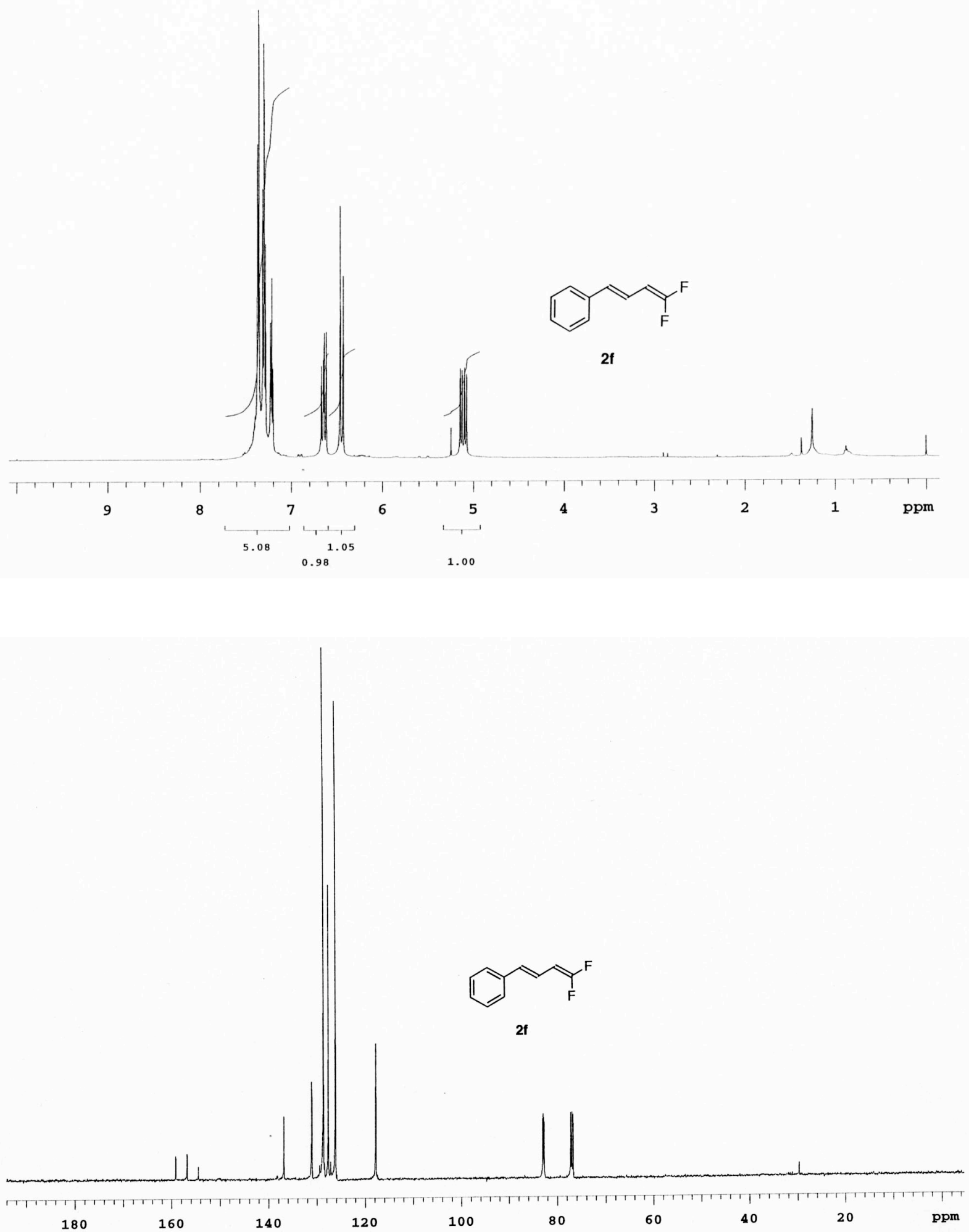

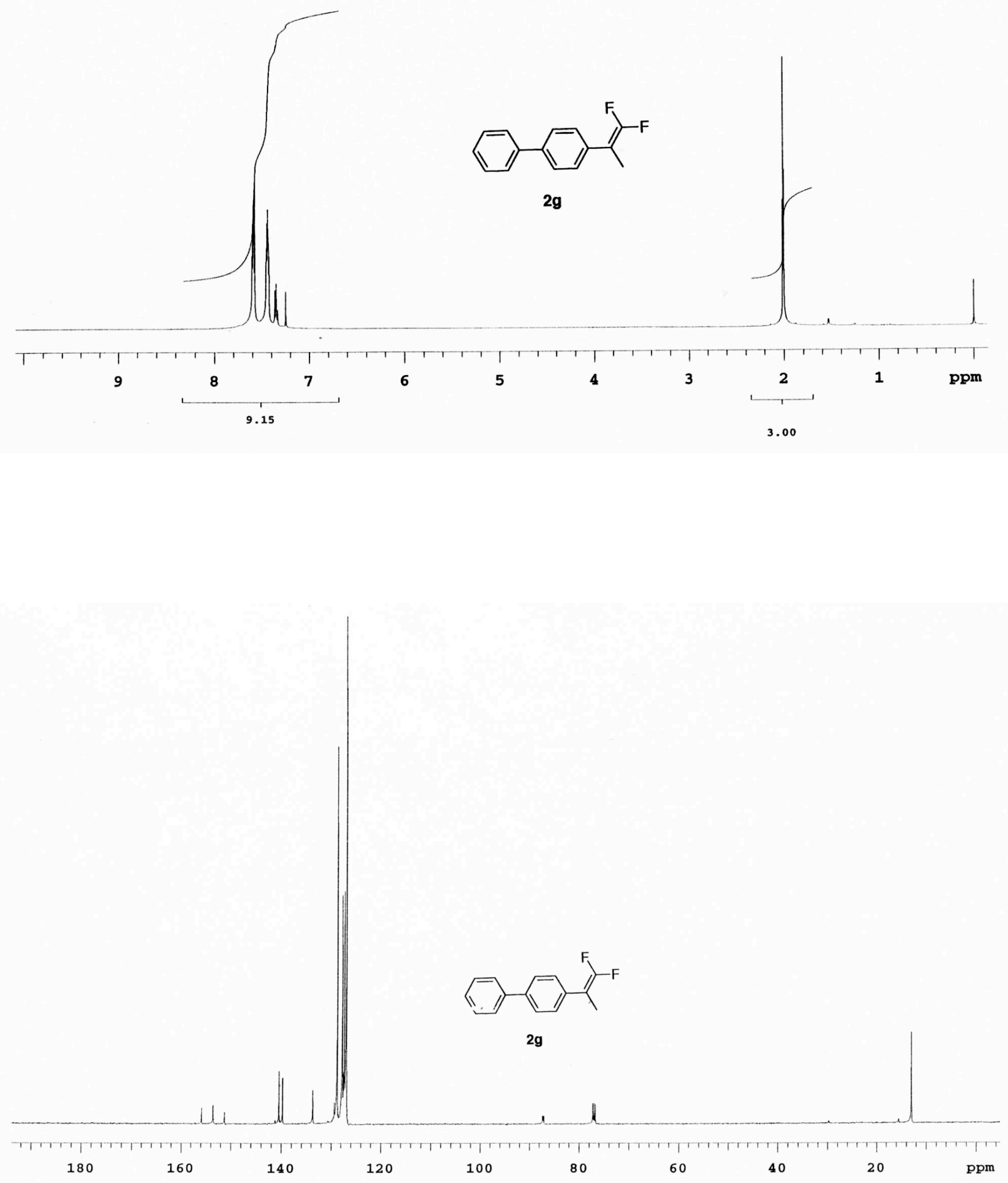

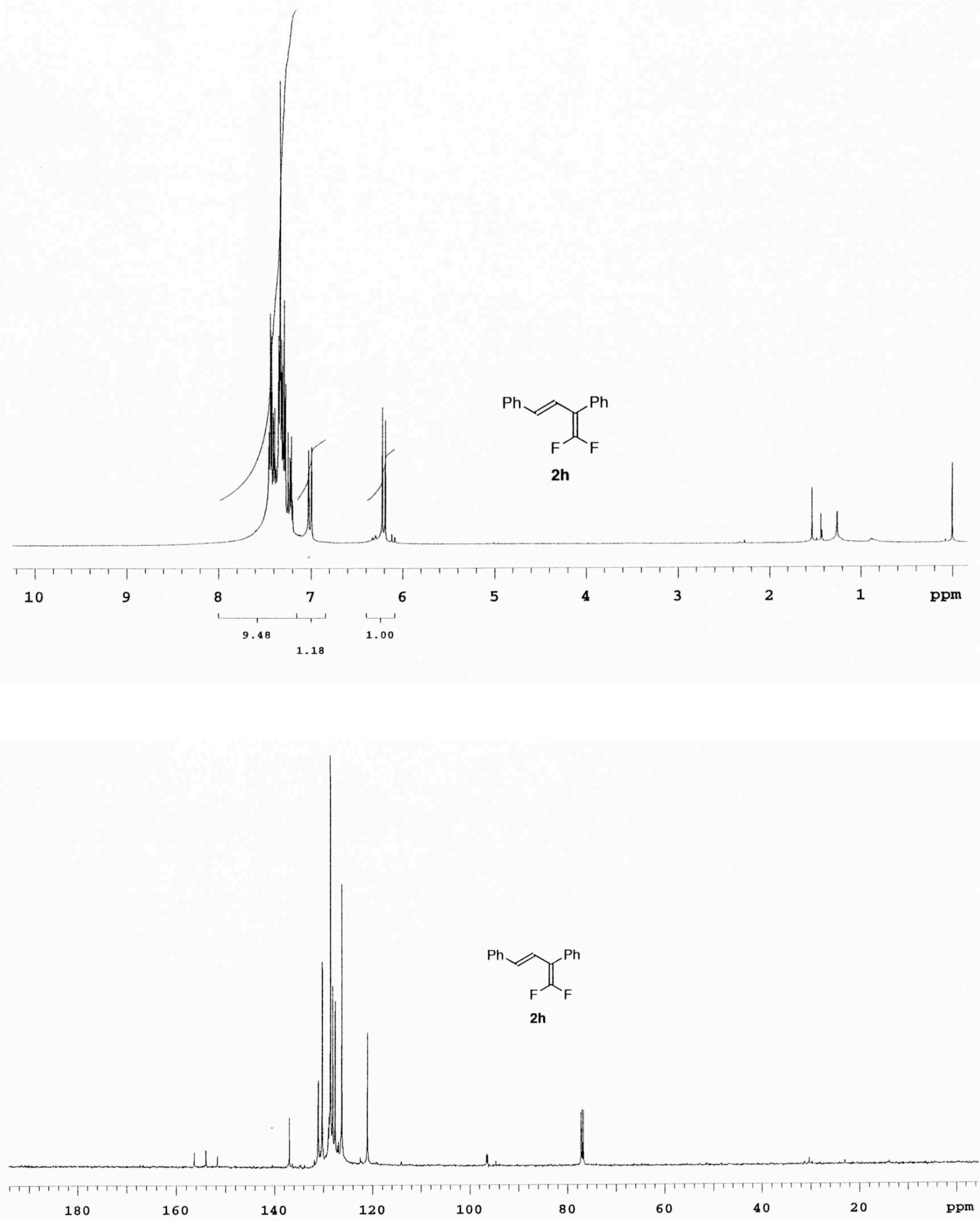

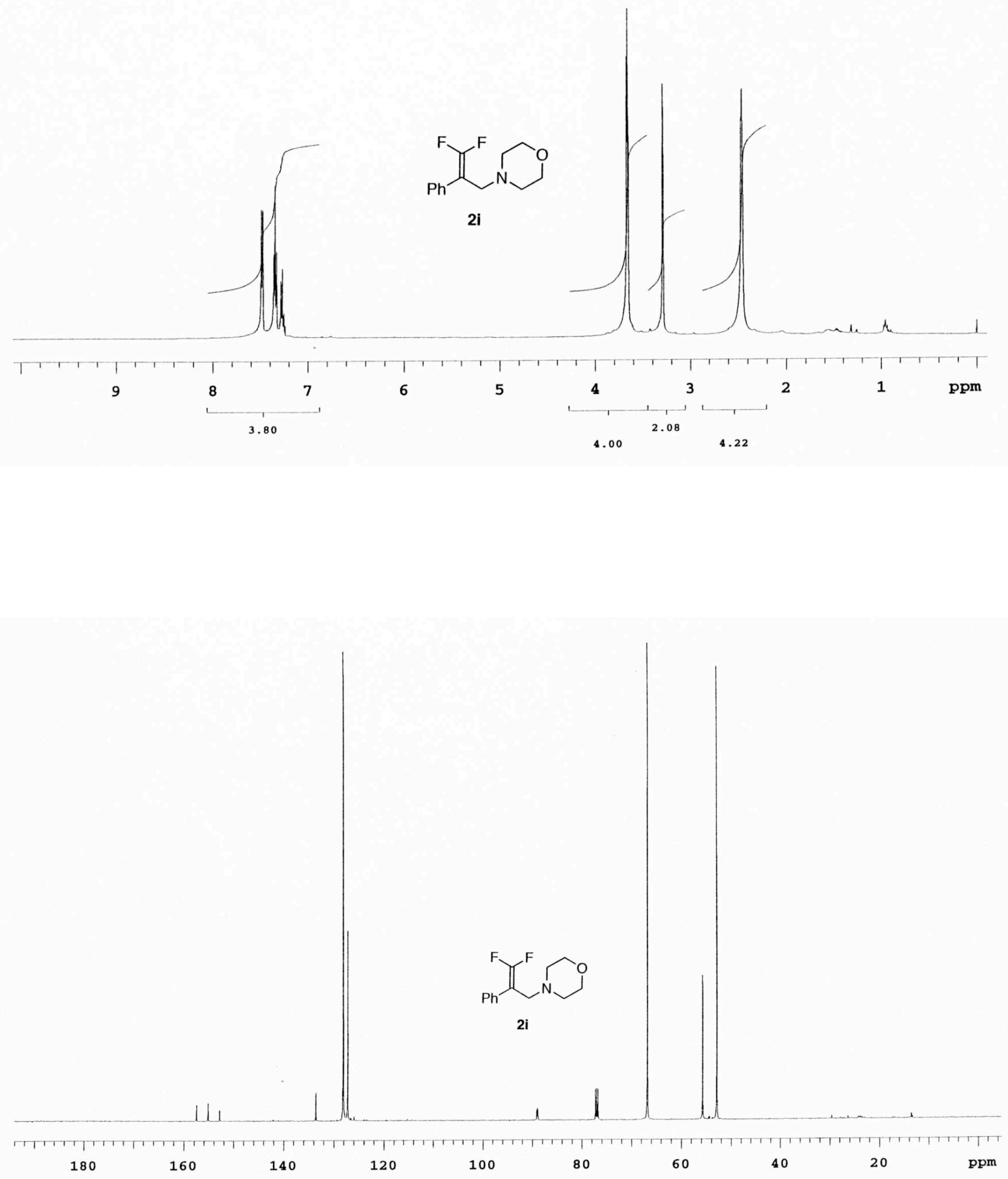

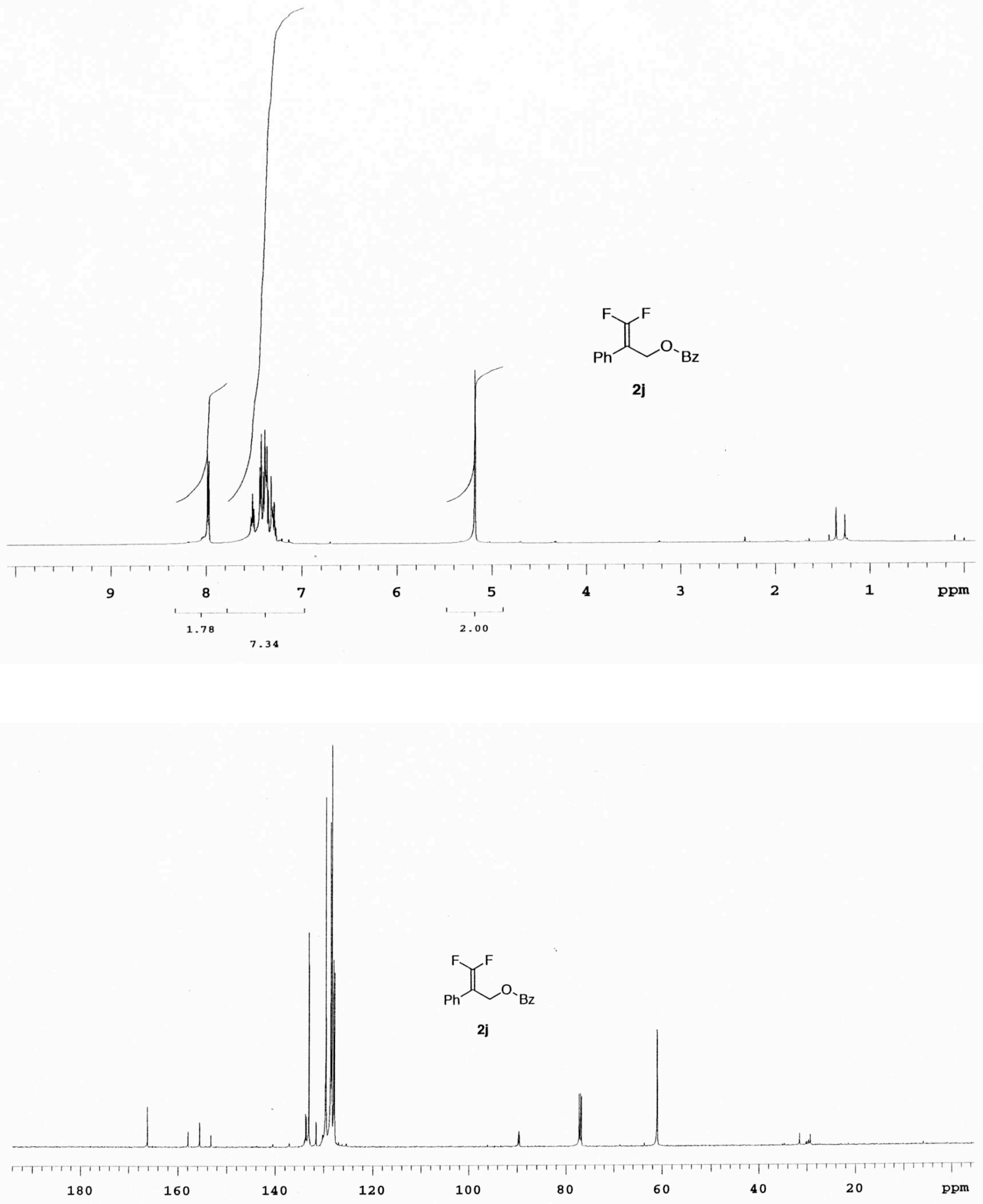


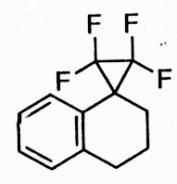

$3 a$
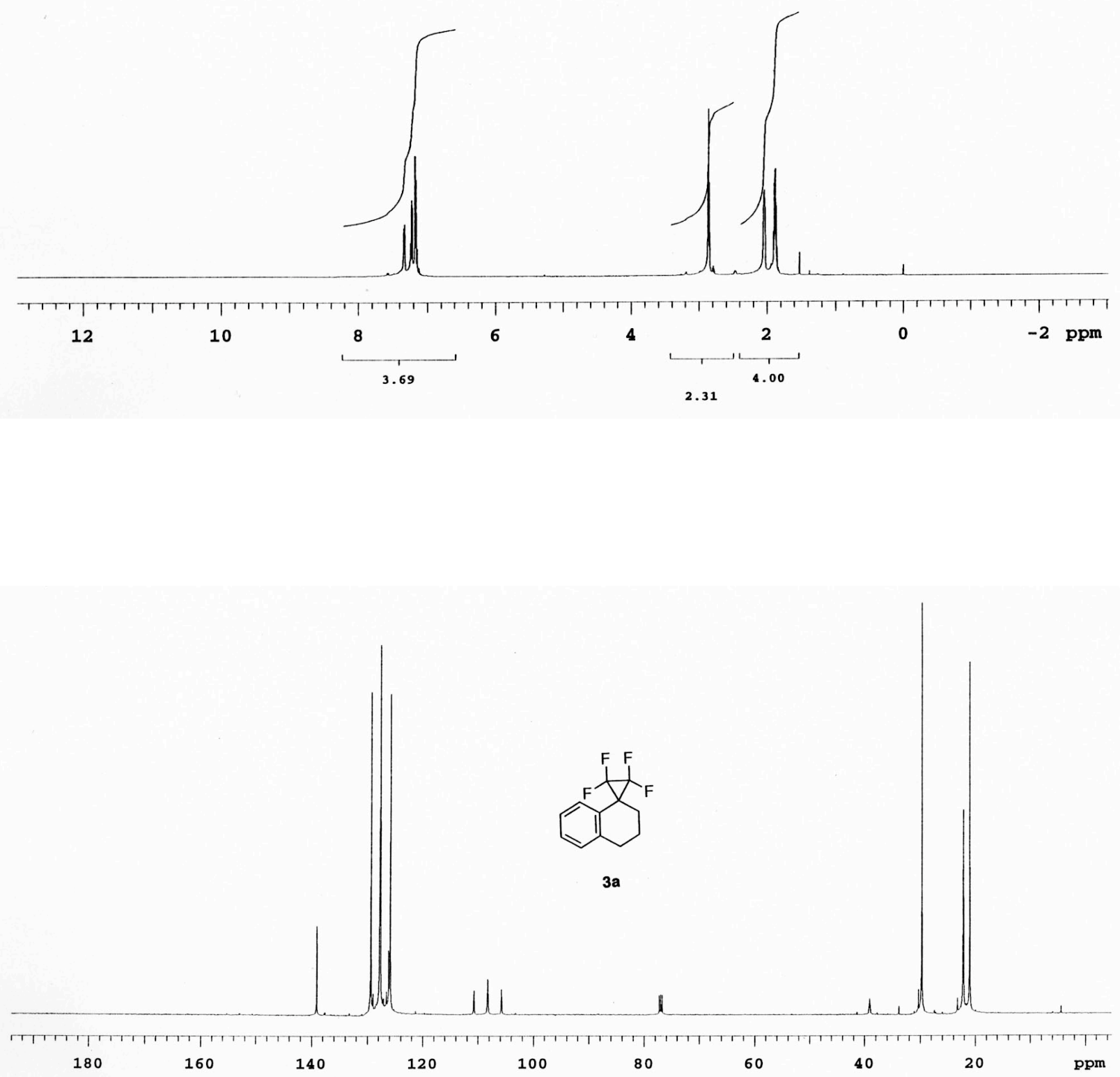

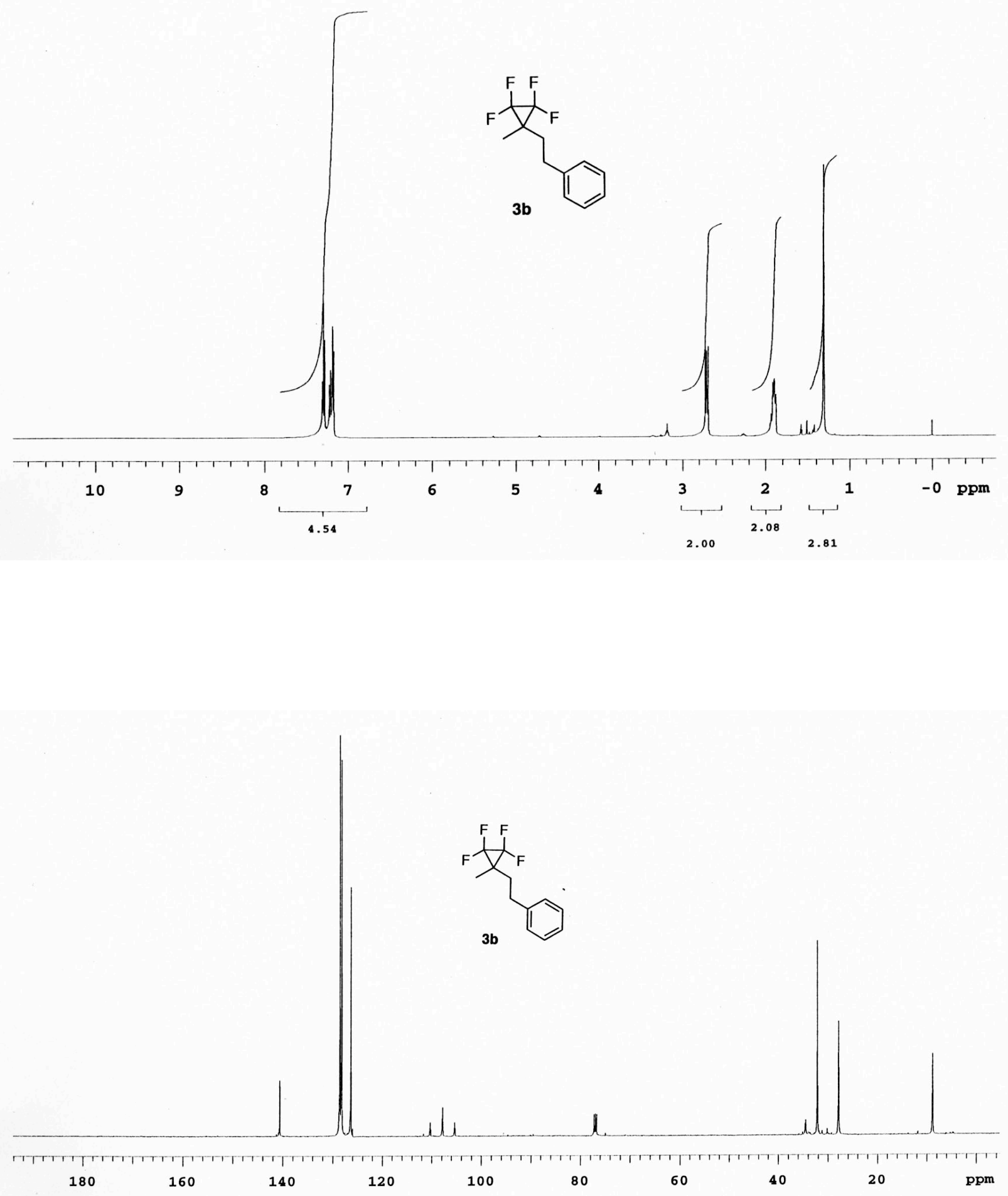
S19
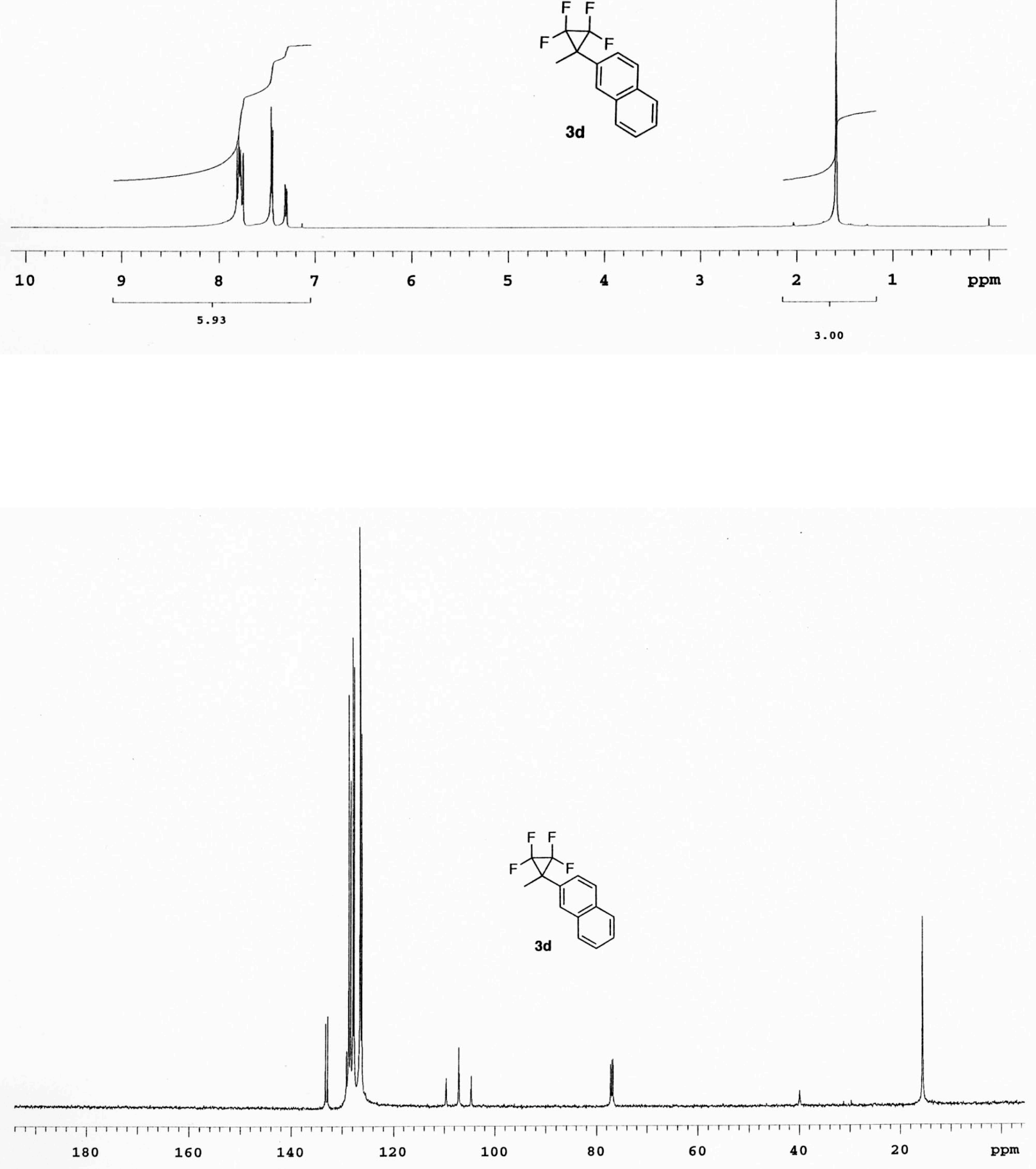
S20
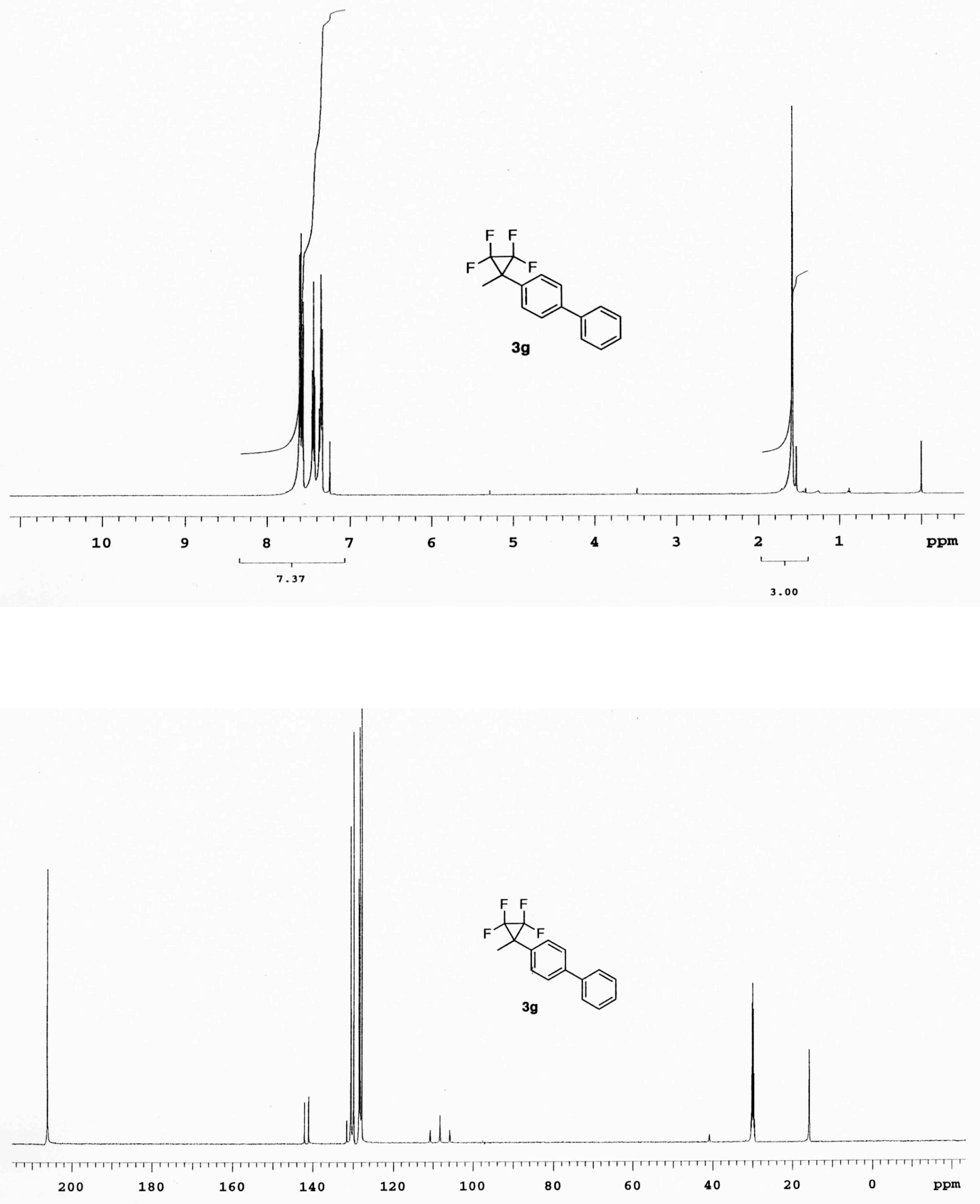

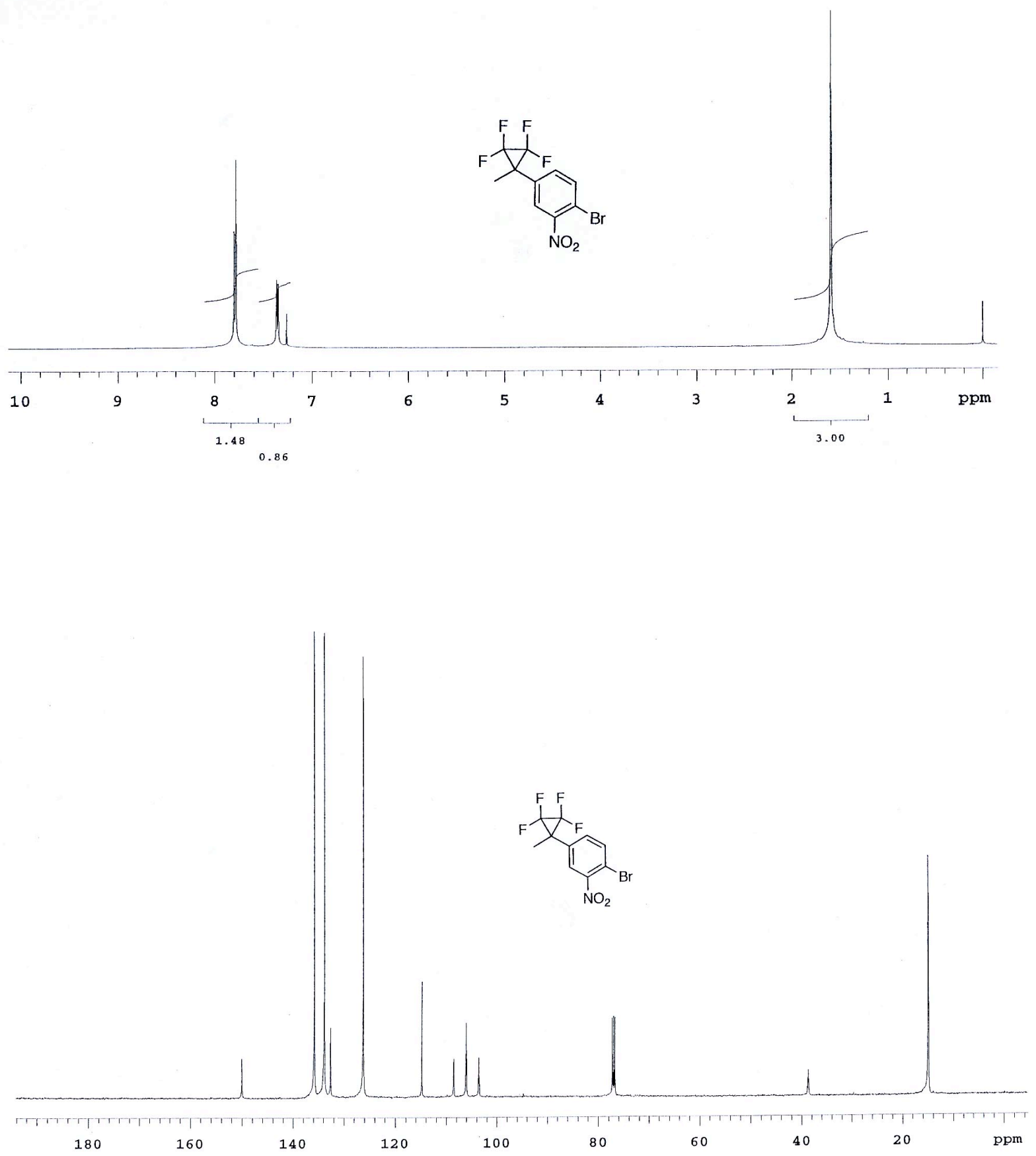


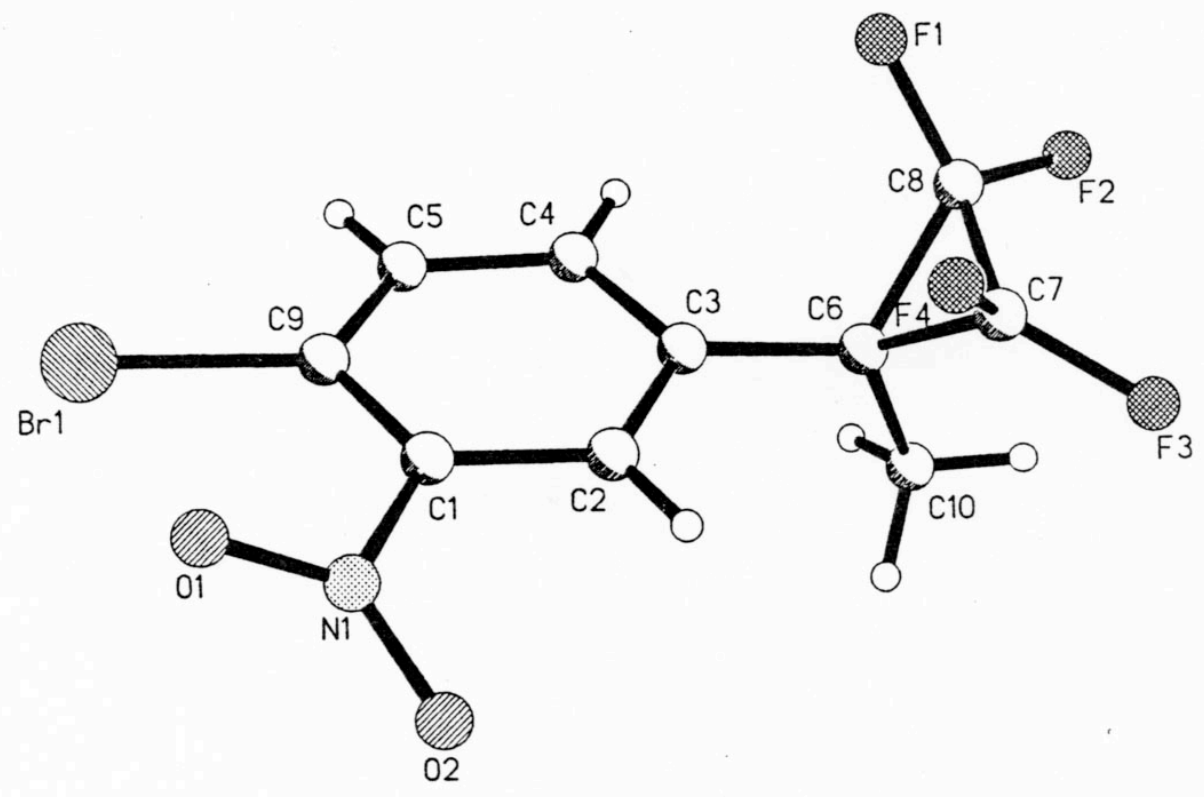

Structure of 1,1,2,2-Tetrafluoro-3-(4-bromo-3-nitrophenyl)-3-methylcyclopropane 